\title{
Increasing emergence of antibacterial resistance mainly in uropathogens: southeast part of India
}

\author{
Bhargavi P.S. ${ }^{1}$, Gopala Rao T.V. ${ }^{2}$, Mukkanti K. ${ }^{3}$, Dinesh Kumar B. ${ }^{4}$, Krishna T.P. ${ }^{4}$ \\ ${ }^{1}$ Montessori Mahila Kalasala, Vijayawada, Andhra Pradesh, India \\ ${ }^{2}$ Gandhi Institute of Biological Sciences, Gunpur, Orissa, India \\ ${ }^{3}$ Department of Environmental Sciences, J.N.T.U, Kukatpally, Hyderabad, Andhra Pradesh, India \\ ${ }^{4}$ NIN, Hyderabad, Andhra Pradesh, India
}

\begin{abstract}
The existence of large concern over the increasing resistance to antibiotics for clinically important pathogens which cause varied number of diseases has led to monitor the prevailing resistance pattern of microbes in a study which was carried out in the city of Vijayawada in Andhra Pradesh. In this study, the data of urinary isolates which showed antibiotic resistance patterns were collected from government hospital, multispecialty hospital and a private laboratory. Total 1424 retrospective samples were collected and evaluated for antimicrobial resistance. The samples $676(47.5 \%)$ were positive and $748(52.5 \%)$ were negative to the infection. The antibiotics which showed higher rate of resistance by the urinary isolates were Amoxicillin, Ciprofloxacin, Co-Trimoxazole, Norfloxacin, Nalidixic acid. In contrast, organisms showed susceptibility to Amikacin and then to Azithromycin. Among the gram negative organisms, Escherichia coli was resistance to Amoxicillin (100\%), Nalidixic Acid (92.6\%), while Klebsiella pneumoniae was found resistance to Amoxicillin (96.3\%), Gentamycin (83.3\%), Pseudomonas aeruginosa was highly resistance $(100 \%)$ to Amoxicillin, Ciprofloxacin, Norfloxacin, Nalidixic acid and Co-trimoxazole. Under gram positive organisms Staphylococcus aureus showed resistance to Amoxicillin (72.7\%), Nalidixic acid (66.7\%) and Enterococcus faecalis was resistance to Nalidixic acid (88.9\%) and Norfloxacin (83.3\%).
\end{abstract}

Key Words: antibiotics, resistance, biological samples, gram negative and gram positive organisms.

\section{Introduction}

Antibiotics are the important medicaments which are used to kill/inhibit the growth of microorganisms which cause many infections (pneumonia, tuberculosis, gonorrhea, throat infections, typhoid etc.,) by their metabolic activities in human beings. Though the antibiotics are important, their inappropriate and large usage is causing the organisms to develop resistance towards the antibiotics, leading to delayed treatment to infections. So, this makes finding emerging antibiotics to fight against old diseases (because resistant strains of bacteria have emerged for resistance towards primary generation of antibiotics) and a parallel problem to find out next generation of antibiotics to fight against new diseases [1]. Antibiotic resistance occurs when an antibiotic has lost its ability to kill bacterial growth, in other words, the bacteria are resistant and continue to multiply in the presence of therapeutic levels of an antibiotic. The emergence and increasing pattern of antimicrobial resistance became a challenge to public health [2]. Poverty, inadequate access to drugs, increased use and misuse are major forces in the development of resistance [3]. Despite the plenty of progress in the antimicrobial chemotherapy, bacterial pathogens gradually develop resistance. Even the use of drug combinations has resulted in making microbial resistance to antibiotics. The first bacterium to show resistance was "Staphylococcus aureus", which causes pneumonia. It becomes resistant to penicillin within four years of its use by altering its cell wall. Haphazard use of antibiotics may also make the bacteria resistance to them [4].

\section{Materials and Methods}

This is a retrospective survey study of culture sensitive records for a period of one year at Government Hospital Laboratory (GHL), Multispeciality Hospital Laboratory (MHL) and a Private pathological Laboratory (PL). The culture sensitivity tests were performed by Kirby Bauer method by using antibiotic discs. A total of 1424 samples records were collected. Out of them 676 were positive (+ve) and 748 were negative (ve), 388 were males and 288 were females among positive cases. The collected data was coded, computerized and analyzed by SPSS [Statistical Package for Social Sciences] Software version 14.6.

Results

The infection profile based on culture sensitive reports at different health care units such as Government Hospital Laboratory (GHL), Multispeciality Hospital Laboratory (MHL) and a Private pathological Laboratory (PL) have been taken for comparison. The highest numbers of samples were recorded from Government unit (GHL) of 547 which contained about $46.8 \%$ of positive to infection, while at Multispeciality hospital laboratory 430 samples were recorded, of them $40.7 \%$ were positive to infection. In the Private pathological laboratory about 447 samples were collected and it contained $48.8 \%$ samples positive to infection.

The data collected for a period of one year contained six (6) of the common human samples viz urine, stool, blood, sputum, throat swab, pus. In Figure.2, an infection profile in various 
pathological samples can be observed. 547 urine samples were recorded which showed $45.5 \%$ positive for the presence of infection caused by organisms and $54.5 \%$ showed negative which is the absence of organisms. Next to urine sample is 364 pus samples which contained $60.2 \%$ positive and $39.8 \%$ negative infection. Of 220 blood samples $21.8 \%$ showed positive and $78.2 \%$ showed negative to infections. The sputum samples of 124 were with $40.3 \%$ positive and $59.7 \%$ negative infection, and lastly 28 throat swab samples which were recorded with $71.4 \%$ positive infection profile and $28.6 \%$ negative to infection profile. Out of the 612 infected positive samples, 250 (40.8\%) Urine samples, $210(34.3 \%)$ pus samples, 48 (7.8\%) sputum samples, $48(7.8 \%)$ stool samples, 37 $(6.0 \%)$ blood and $19(3.1 \%)$ throat swab samples were recorded at different areas of the Vijayawada city. The above mentioned infected human samples laboratory reports showed the presence of many organisms, out of which five (5) different species were found to be more commonly identified at all the areas. Out of total $45.5 \%$ (positive) urine sample, commonest bacterial isolate was $\mathrm{E}$. coli with $49 \%$ and least bacterial isolate was $P$. aeruginosa $4 \%$. The rest were between $10 \%$ and $23 \%$ as shown in fig.-.2. The positive culture reports showed varied responses to different antibiotics by the organisms in the biological samples. Since urinary tract infections are the most common among the infections, frequent and primary testing of urine sample is advised by the practitioners. So this enrouted for more collection and testing of urine samples for the susceptibility pattern of antibiotics. Thus, the study concentrates on the antibiotic susceptibility of urinary isolates. But to surprise high percentage of resistance is shown towards all most all antibiotics by the organisms. Overall there were about 56 different types of antibiotics used to carry out the culture sensitive tests in different areas. Out of these antibiotics the highest frequency of antibiotics exhibiting resistance and sensitivity together was evaluated (Figure.3). The resistance percentage for the antibiotics was higher than the sensitive percentage except for Amikacin and Azithromycin of $75 \%$ and $58.5 \%$ respectively. Amoxicillin, Nalidixic acid, Cotrimoxazole, Ciprofloxacin, Norfloxacin, etc are having higher resistance percentage from $70 \%$ to $90 \%$. It is a known fact, that Escherichia coli is the commonly isolated organism among the urine samples. The other organisms found to be isolated also were Klebsiella pneumoniae, Staphylococcus aureus, Enterococcus faecalis and Pseudomonas aeruginosa. As per the information of Table.1, the resistance and sensitive patterns of gram negative urinary isolates are observed. Ciprofloxacin, Amoxicillin, Co- trimoxazole, Norfloxacin and Nalidixic acid were observed to be $100 \%$ resistance by $P$. aeruginosa. While $E$. coli and $K$. pneumoniae showed $100 \%$ and $96.3 \%$ resistance to Amoxicillin respectively. Amikacin and Azithromycin, showed low resistance (between $20.8 \%$ and $34.8 \%$ respectively) and high sensitivity $(79.2 \%$ and $65.2 \%$ respectively) by $E$. coli, while $K$. pneumoniae was susceptible to Amikacin (85.7\%) only. The other antibiotics such as Ceftazidime, Kanamycin, Gentamycin, Norfloxacin, etc showed resistance rates from $50 \%$ to $85 \%$. Table.2 indicated the resistance and sensitivity of gram positive organisms. $S$. aureus showed high resistance amoxicillin $(72.7 \%)$ and showed sensitivity to Amikacin $(62.5 \%)$ only. E. faecalis showed the resistance rates to be high for Nalidixic acid $(88.9 \%)$ and Norfloxacin (83.3\%). Similar to E. coli, E. faecalis was sensitive to Amikacin (71.4\%) and Azithromycin (80\%). The rest of the antibiotics such as Kanamycin, Ciprofloxacin, Ceftazidime etc., have shown resistance rate from $50 \%$ to $75 \%$ for gram positive organisms.

\section{Discussion}

The study was formulated for an outlay of the increasing trends of antibiotic resistance from the clinical sources at specified areas. Various studies on antibiotic resistance are emerging with an intention to bring into lime light about the resistance that is developing among the microorganisms towards the antibiotics. Though the approaches are same, the results are showing entirely different conclusions from time to time. Organisms are showing different responses between old generation and new generation antibiotics, with a change in their genes of both chromosomal and extra chromosomal, which are the causes for them to show resistance to old generation antibiotics and susceptible to newer generation antibiotics. However, reasons for the cause of this resistant activity can be framed out as either more frequent and unnecessary usage of antibiotics or prescribing newer antibiotics with newer combinations for faster recovery of infections. Urinary tract infections UTI is the most frequent clinical manifestation after respiratory tract infections. It has been observed that $E$. coli is the sole causative agent in more than $80 \%$ of uncomplicated UTI [5]. E. coli [6, 7, and 8] and Klebsiella [9] have been reported as the most common organisms causing UTI. In the present study, the top resistance percentage was observed by Amoxycillin (89.6\%), Nalidixic acid (86.7\%), Co-Trimoxazole (77.3\%), Ciprofloxacin (73.4\%), Norfloxacin (73.2\%). There are studies conducted nationally and internationally regarding the emerging trend in antibiotic resistance. Studies conducted in Eastern Jorden was similar to the present study in organism profile in urine sample and resistance pattern 
was high to Co-trimoxazole (59.5\%), Gentamycin $(43.2 \%)$, Nalidixic acid (29.3\%) and least to Norfloxacin (11\%) and Ciprofloxacin (3.8\%) [10]. In another study in Saudi Arabia showed increase in antibiotic resistance between 1999 and 2002 for Ciprofloxacin (between 2\% and $35 \%$ ), Gentamycin (between $2 \%$ and $33 \%$ ), Ceftazidime (between $10 \%$ and $15 \%$ ) by E. coli, $K$. pneumoniae and P.aeruginosa [11]. While another study in same Saudi Arabia showed sensitiv patterns for Norfloxacin and Nalidixic acid with similar organism profile to that of the present study [12]. A 15 year reviewed data at Netherlands showed gradual decrease in susceptibility patterns for Co-trimoxazole (from $79 \%$ to $62 \%$ ), Norfloxacin (from $87 \%$ to $71 \%$ ) by E. coli, while K.pneumoniae showed $70 \%$ resistance to Norfloxacin and $69 \%$ resistance to Co-trimoxazole and $P$. aeruginosa was $47 \%$ resistance to Norfloxacin.Gentamycin was resistance by Klebsiella and Psedomonas but showed susceptibility by E.coli [13]. E. coli showed increased resistance to Norfloxacin and was less resistant to Amoxicillin in an Italian multicentre survey [14]. Another observation in a retrospective study conducted in Iran showed high resistance to Co-trimoxazole by $E$. coli (26.3\%) and K.pneumoniae (54.5\%), but very least resistance percentage to amikacin (5\% to $10 \%$ ) by $E$. coli and $K$. pneumoniae while gentamycin was less resistant by $E$. coli $(5.25 \%)$ and highly resistant by $\mathrm{K}$. pneumonia [15]. Studies conducted in India also showed almost similar findings. A study at Kashmir showed more susceptibility to Amikacin and very least susceptibility to Co-trimoxazole by $E$. coli and $K$. pneumoniae [16]. A retrospective study in a tertiary care setting in Bangalore had high resistance to Co-trimoxazole, Amicillin, Amoxicillin and fluroquinolones by $E$. coli [17]. Similar to the present study at Aligarh, Cotrimoxazole topped the resistance pattern (between 40\% and 100\%), and Amikacin showed the least resistance percentage (between $20 \%$ and $51 \%$ ) by E.coli, $K$. pneumoniae, S.aureus and $P$. aeruginosa. While Ciprofloxacin and Norfloxacin showed resistance by the isolates from $30 \%$ to $70 \%$ and Gentamycin was resistance from $20 \%$ to $70 \%$ [18]. In a study in New Delhi topped high resistance patterns of Gentamycin (57.1\% to $90 \%$ ) among the other antibiotics, when compared to the present finding of $40 \%$ to $80 \%$. Amikacin showed least resistance when compared to other antibiotics. In contrast to the present study Norfloxacin showed suscpetibility by $P$. aeruginosa, $E$. faecalis and $S$. aureus, while high resistance was observd by gram negative and gram positive organisms [19]. A study in Manipur showed similar finding to the present study an, where $P$. aeruginosa showed $100 \%$ resistance to Cotrimoxazole and a contrast finding of sensitivity to
Norfloxacin (72.3\%). Another contrast observation was sensitivity to Gentamycin by E.coli [69.5\%] and $K$. pneumoniae (76.7\%), Sensitivity to Co-trimoxazole $(65.5 \%)$ and Ciprofloxacin (60.8\%) was observed by $K$. pneumoniae [20]. In a study of antibiotic sensitivity and resistance in urinary isolates in Khatmandu valley showed complete contrast observation to the present study where E.coli, $K$. pneumoniae and $S$. aureus were showing sensitive pattern to Amoxicillin (between 64\% and $89 \%$ ), Norfloxacin (between $65 \%$ and $75 \%$ ), Gentamycin (between 90\% and 93\%) and Cephalosporins (upto 89\%). Amikacin was showing susceptibility from (between $80 \%$ and $100 \%$ ) by all the isolates (including $P$. aeruginosa). Report shows that except for Amikacin, P.aeruginosa was resistance to all the antibiotics [21]. Studies have already showed that Nalidixic acid, Norfloxacin and Ciprofloxacin were least effective against all the uropathogens [22, 23]. Further, a study at Nagpur, $82 \%$ and $79.6 \%$ of urinary isolates to be resistant to cotrimoxazole and ampicillin, respectively indicating maximum resistance to these drugs [24].

\section{Conclusion}

The present findings are quite alarming to find almost all antibiotics are showing high percentage of resistance by the urinary isolates. So, this infromation not only relevant for the local area but useful as regional data of reffernce for the physicians. Briefly, it can be concluded that based on the highlightings of few observations in a year long survey in Vijayawada on microorganisms which are not responding alike as the same towards antimicrobial agents at all the places. This antibiotic resistance could have developed due to irrational use of antibiotics, and insufficient, irregular use of antibiotics to infections which are not properly diagnised. Further, it is also because of not a proper consumption of new generation antibiotics for the faster recovery from infections. The organisms show resistance to antibiotics that were susceptible to the same antibiotics at the same place of survey. New Drug combination therapy of antibiotics may be followed against organisms. These all facts for the development drug therapy will make awareness in the patients for following laboratory based reports and for prescriptions with the help of doctors. It is also necessary that the patients may always follow the doctors prescriptions rather than their self treatments.

\section{Acknowledgements}

The authors are very much thankful to NIN director for the discussions with their scientists throuhout this work. In addition thankful to GIBS, Gunupur -765022 for the platform of this work. 


\section{References}

[1] Kenneth Todar (2008) In a book Todar's online text book of bacteriology. http://www.textbookof bacteriology.net/resantimicrobial_4.html

[2] About Antibiotic resistance. http://www.tufts.edu/med/apua/Q\&A/Q\& A_AR.html.

[3] Kamini W. (2006) Emerging Problem of Antimicrobial Resistance in Developing Countries: Interwining Socio economic Issues. Regional Health Forum WHO South- East Asia Region, 7( 1), 2006.

[4] Vibha V. Broad spectrum drug rule India. http://www.downearth.org.in/full6.

[5] Rao B.L.N., Gowal D., Chaturvedi A.P., Jayasheela M., and Agarwal P. (1987) Indian J Med Microbiol. 7, 21-2.

[6] Khurana S., Taneja N., Sharma M. (2002) Indian J Med Res., 116, 145-149

[7] Gupta V., Yadav A. and Joshi R.M. (2002) Indian J Med Microbiol. 20, 96-98.

[8] Gales A.C., Sader H.S., Jones, R.N. (2002) Diagn. Microbial Infect. Dis. 44, 289299.

[9] Bajaj J.K., Karyokart R.P., Kulkarni J.D. and Deshmukh A.B. (1999) J. Commun. Dis. 31, 181-184.

[10] Younis N., Quol K., Momani T, Awaisheh F.A. and Kayed D.A. (2009) J. Nepal Med. Assoc., 48(173): 14-19.

[11] Abdulrahman A. K., Kumar A. and Dass S.M. (2004) Saudi J. Kidney Dis Trans.15(2), 135-139.

[12] Ahmad S. and Ahmad F. (1995) Bangladesh Med. Res. Coun. Bull.. 21 (3): $95-98$.

[13] Fluit A.C., Mark E., Jones E., Schmitz,F.J., Acar J. and Gupta R. (2000) Antonie van Leeuwenhoek 77: 147-152.

[14] Schito G.C., Chezzi C., Nicoletti G., Moreddu M., Arcangeletti M.C., Stefani S., Albini E., Bono G.P.D. (1992) Infect. 20 (4). 291.

[15] Movahedian A.H., Mosayebi Z., Moniri S. (2007) J. Infect. Dis. Antimicrob. Agents. 24, 7-11.

[16] Manzoor S., Gash K. and Rukhsana B.A. (2004) Ind. J. for the Practising Doc.1, (1), 23-27.

[17] Priya P., Khurana R. and George J. (2002) Indian J. Pharma., 34; 278-280.

[18] Mohammed A., Mohammed S., Asad and Khan U. (2007) Ind. Annals of Clin. Microbiol. \& Antimicrob., 6:4-6.

[19] Hasan A.S., Nair D., Kaur J., Baweja G., and Deb P. (2007) J Ayub Med Coll Abbottabad ., 19 (1). 21-24.
[20] Yengkokpam C., Ingudam D., Yenngkokpam I.S. and Jha B.K. (2007) Nepal Med. Coll. J., 9 (3): 170-172.

[21] Jha N. and Bapat S.K. (2005) Khatmandu Univ. Med. J., 3 No.2, Issue 10, 123129.

[22] Elliot T.S., Shelton A. and Greenwood D. (1987) J Med Microbiol., 23:83-88.

[23] Shobha R., Gupta R. and Gaheer R. (2000) Indian J Med Sci., 54:388-94.

[24] Supriya S., Suresh T., Jalgaonkar V., Ahamad S. and Hassani U. (2008) Indian J Med Res 120, 553-556. 


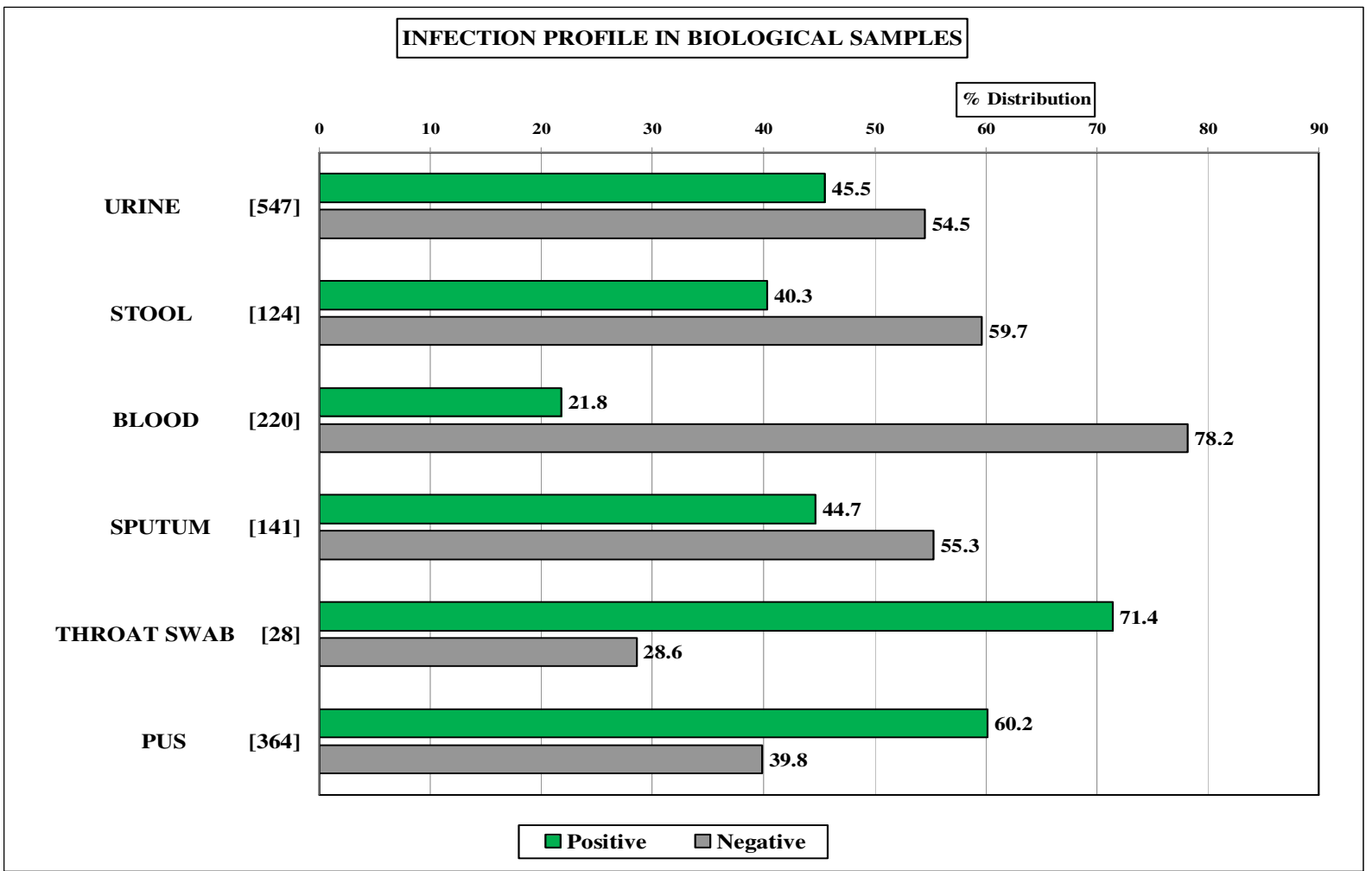

Fig. 1- Percentage distribution of infection profile in 1424 human biological samples collected from a Survey of culture sensitivity reports

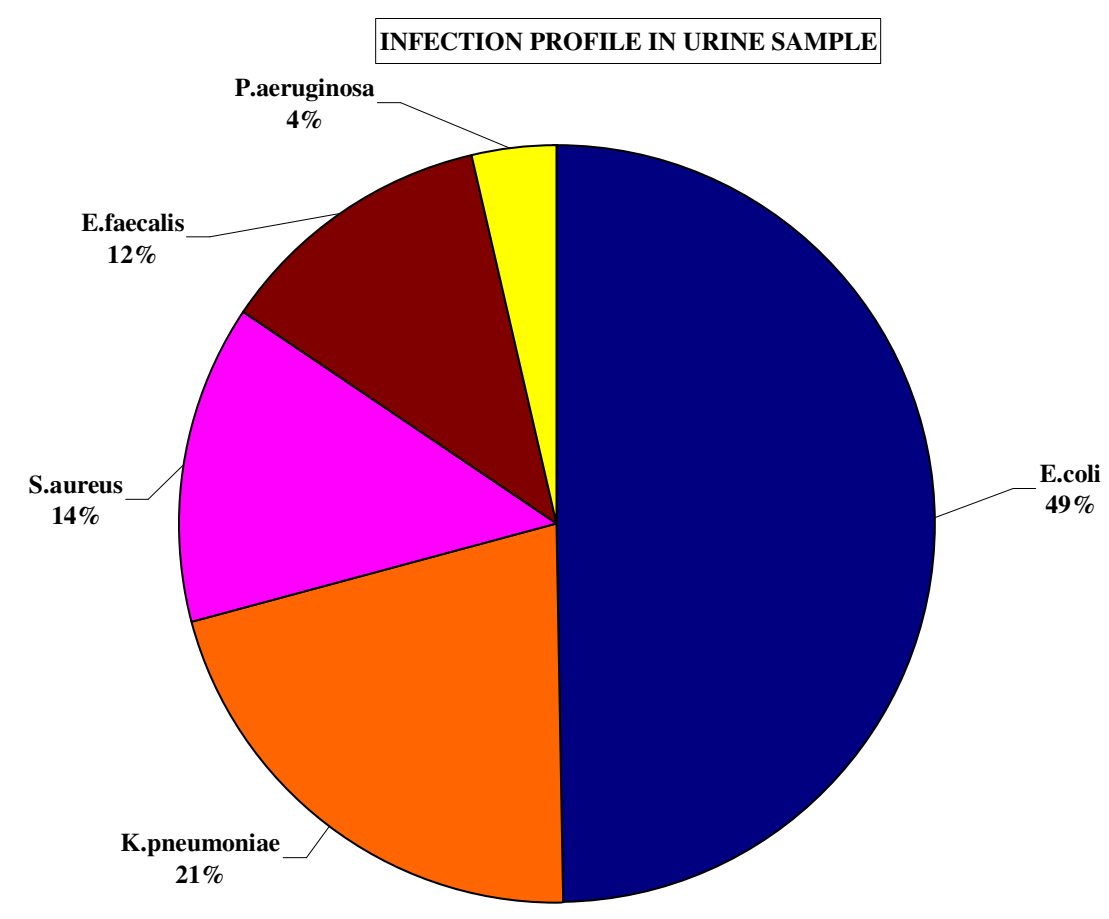

Fig. 2- Profile of organisms in the positive biological samples collected and Individual organism profile in biological samples collected from the culture sensitivity reports. 


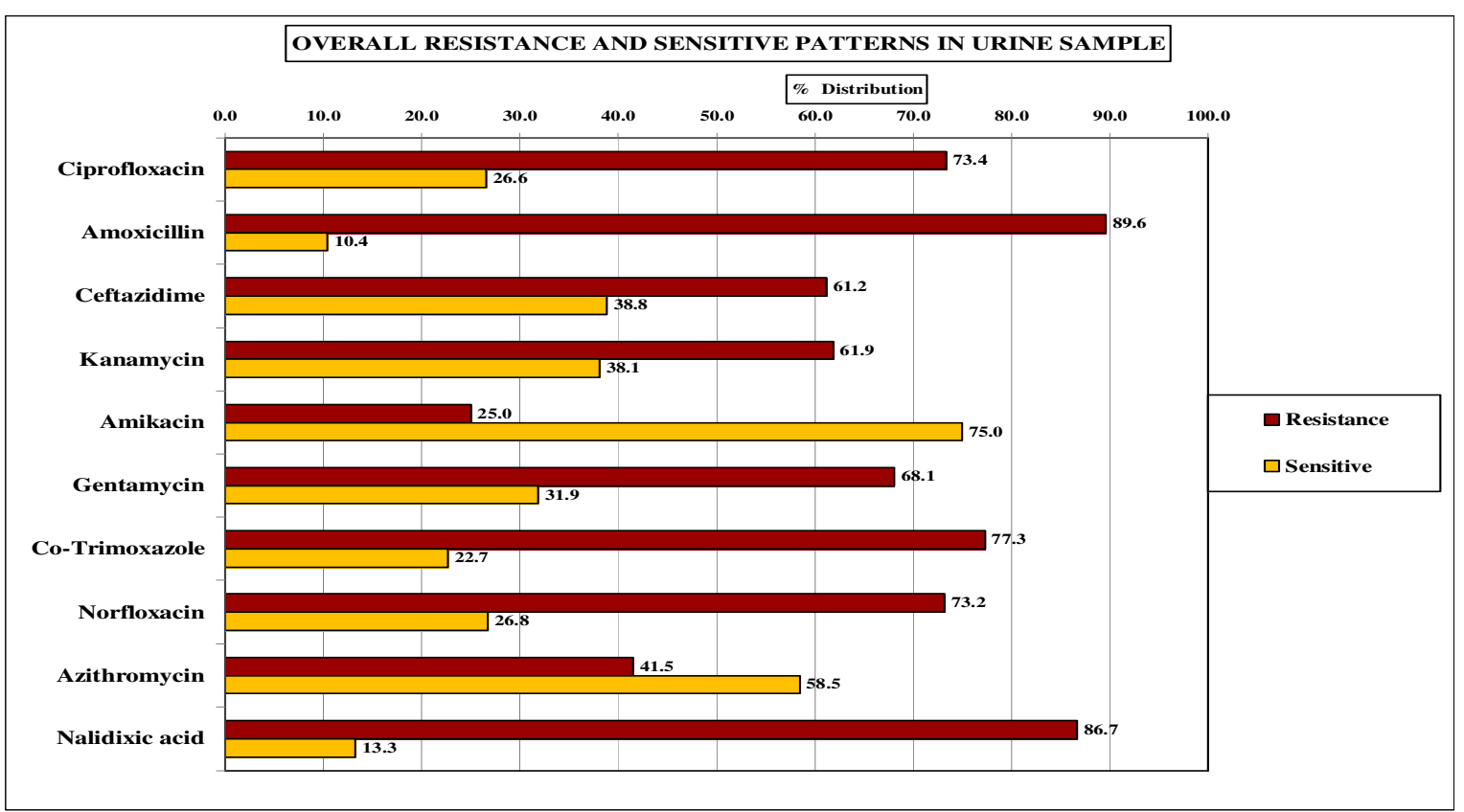

Fig. 3- Antibiotic profile showing overall resistance and sensitive percentage patterns in urine samples.

Table 1- Gram Negative organisms showing resistance and sensitivity to antibiotics in urine sample

\begin{tabular}{|l|l|l|l|l|l|l|}
\hline \multirow{2}{*}{ Antibiotics } & E. coli & \multicolumn{2}{|l|}{ K. pneumoniae } & \multicolumn{2}{|l|}{ P. aeruginosa } \\
\cline { 2 - 7 } & $\begin{array}{l}\text { Resistance } \\
\%\end{array}$ & $\begin{array}{l}\text { Sensitivity } \\
\%\end{array}$ & $\begin{array}{l}\text { Resistance } \\
\%\end{array}$ & $\begin{array}{l}\text { Sensitivity } \\
\%\end{array}$ & $\begin{array}{l}\text { Resistance } \\
\%\end{array}$ & $\begin{array}{l}\text { Sensitivity } \\
\%\end{array}$ \\
\hline Ciprofloxacin & 91.9 & 8.1 & 58.6 & 41.4 & 100.0 & 0.0 \\
\hline Amoxicillin & 100.0 & 0.0 & 96.3 & 3.7 & 100.0 & 0.0 \\
\hline Ceftazidime & 60.0 & 40.0 & 57.7 & 42.3 & 0.0 & 0.0 \\
\hline Kanamycin & 62.5 & 37.5 & 57.1 & 42.9 & 80.0 & 20.0 \\
\hline Amikacin & 20.8 & 79.2 & 14.3 & 85.7 & 50.0 & 50.0 \\
\hline Gentamycin & 61.5 & 38.5 & 83.3 & 16.7 & 80.0 & 20.0 \\
\hline Co-Trimoxazole & 78.1 & 21.9 & 82.4 & 17.6 & 100.0 & 0.0 \\
\hline Norfloxacin & 66.7 & 33.3 & 82.4 & 17.6 & 100.0 & 0.0 \\
\hline Azithromycin & 34.8 & 65.2 & 57.1 & 42.9 & 66.7 & 33.3 \\
\hline Nalidixic acid & 92.6 & 7.4 & 80.0 & 20.0 & 100.0 & 0.0 \\
\hline
\end{tabular}

Table 2- Gram positive organisms showing resistance and sensitivity to antibiotics in urine sample

\begin{tabular}{|l|l|l|l|l|}
\hline \multirow{2}{*}{ Antibiotics } & \multicolumn{3}{|l|}{ S. aureus } & \multicolumn{2}{l|}{ E. faecalis } \\
\cline { 2 - 5 } & $\begin{array}{l}\text { Resistance } \\
\%\end{array}$ & $\begin{array}{l}\text { Sensitivity } \\
\%\end{array}$ & $\begin{array}{l}\text { Resistance } \\
\%\end{array}$ & $\begin{array}{l}\text { Sensitivity } \\
\%\end{array}$ \\
\hline Ciprofloxacin & 63.6 & 36.4 & 60.0 & 40.0 \\
\hline Amoxicillin & 72.7 & 27.3 & 63.6 & 36.4 \\
\hline Ceftazidime & 60.0 & 40.0 & 71.4 & 28.6 \\
\hline Kanamycin & 55.6 & 44.4 & 63.6 & 36.4 \\
\hline Amikacin & 60.0 & 40.0 & 28.6 & 71.4 \\
\hline Gentamycin & 44.4 & 55.6 & 72.7 & 27.3 \\
\hline Co-Trimoxazole & 62.5 & 37.5 & 69.2 & 30.8 \\
\hline Norfloxacin & 62.5 & 37.5 & 83.3 & 16.7 \\
\hline Azithromycin & 37.5 & 62.5 & 20.0 & 80.0 \\
\hline Nalidixic acid & 66.7 & 33.3 & 88.9 & 11.1 \\
\hline
\end{tabular}

